\title{
Capsule Commentary on Piller et al., Characteristics and Long-Term Follow-Up of Participants With Peripheral Vascular Disease During ALLHAT
}

\author{
Stephen K. Williams, MD, MS \\ Department of Population Health, Center for Healthful Behavior Change, New York University School of Medicine, New York, NY, \\ USA.
}

J Gen Intern Med 29(11):1527

DOI: $10.1007 / \mathrm{s} 11606-014-2983-\mathrm{x}$

(c) Society of General Internal Medicine 2014

$\mathrm{M}$ ore than a decade ago, the ALLHAT trial ${ }^{1}$ influenced the Joint National Committee (JNC) 7 to recommend diuretics for initial anti-hypertensive therapy in patients without "compelling indications." JNC 8 does not comment on specific high-risk conditions considered compelling indications, other than chronic kidney disease (CKD). ${ }^{3}$ However, there are other conditions generally considered compelling indications to start a specific anti-hypertensive class, including: heart failure, postmyocardial infarction status, high coronary heart disease risk, diabetes and prior stroke. ${ }^{2}$

In this issue, a sub-study of the ALLHAT trial asks the question of whether peripheral artery disease (PAD) could be another compelling indication for choosing a specific antihypertensive class; namely, calcium channel blockers. ${ }^{4}$ In the original ALLHAT publication, ${ }^{1}$ analysis of the individual components of the secondary outcome revealed an intriguing trend for a lesser incidence of PAD among participants started on amlodipine, in comparison to chlorthalidone (Table 5). In the current follow-up paper, using a stricter definition for PAD, an almost identical trend for a lower incidence of PAD was found for amlodipine, though the finding remained not quite statistically significant. Based on the results of this analysis, PAD continues to stand alone as a major high-risk cardiovascular condition without compelling evidence to use a specific antihypertensive agent in order to improve outcomes.

The authors conclude that there is a compelling need for comparative outcome trials examining treatment of PAD in high-risk patients. Unfortunately, this is no easy proposal, since monotherapy is rarely sufficient to adequately control hypertension in this complicated population. For example, most
ALLHAT participants ended up needing a second line antihypertensive agent to achieve goal blood pressure levels. Therefore, we still need to figure out how to adequately account for the confounding that occurs in these multiple agent trials.

In the interim, it should be appreciated that patients with PAD generally would be noted to have other comorbidities that are considered compelling indications for use of certain anti-hypertensive classes. We should use these comorbidities to guide our therapy of these high-risk patients.

Conflict of Interest: The authors declare that they do not have a conflict of interest.

Corresponding Author: Stephen K. Williams, MD, MS; Department of Population Health, Center for Healthful Behavior Change New York University School of Medicine, New York, NY, USA (e-mail: stephen.williams@nyumc.org).

\section{REFERENCES}

1. ALLHAT Officers and Coordinators for the ALLHAT Collaborative Research Group. Major outcomes in high-risk hypertensive patients randomized to angiotensin-converting enzyme inhibitor or calcium channel blocker vs diuretic: The Antihypertensive And Lipid-Lowering Treatment To Prevent Heart Attack Trial (ALLHAT). JAMA. 2002;288:2981-2997.

2. Chobanian AV, Bakris GL, Black HR, et al. The Seventh Report of the Joint National Committee on Prevention, Detection, Evaluation, and Treatment of High Blood Pressure: the JNC 7 report. JAMA. 2003;289:2560-2572.

3. James PA, Oparil S, Carter BL, et al. Evidence-Based Guideline for the Management of High Blood Pressure in Adults: Report From the Panel Members Appointed to the Eighth Joint National Committee (JNC 8). JAMA. 2014;311:507-20.

4. Piller LB, Simpson LM, Baraniuk S, et al. Characteristics and Long-Term Follow-up of Participants with Peripheral Arterial Disease during ALLHAT. J Gen Intern Med. DOI: 10.1007/s11606-014-2947-1 